\title{
The Three Dimensions of Unsolicited Dick Pics: Men Who have Sex with Men's Experiences of Sending and Receiving Unsolicited Dick Pics on Dating Apps
}

\section{Christopher Dietzel ${ }^{1}[0$}

Accepted: 17 October 2021 / Published online: 29 October 2021

(C) The Author(s), under exclusive licence to Springer Science+Business Media, LLC, part of Springer Nature 2021

\begin{abstract}
The unsolicited "dick pic" (DP), which refers to a photo of a penis that is sent without the consent of the recipient, has been identified as a form of technology-facilitated sexual violence (Powell \& Henry, 2017). While men who have sex with men (MSM) experience elevated rates of technology-facilitated sexual violence, much of the research has focused on interactions between heterosexual men and women. This study investigated the experiences that MSM have with sending and receiving unsolicited DPs on dating apps. Analysis of interviews with 25 MSM dating app users in Canada revealed three "dimensions" of unsolicited DPs-consensual, wanted, and typical-that capture users' experiences of receiving such images relative to consent and sexual violence frameworks. Seven factors, including the attractiveness of the sender and the DP, had an impact on MSM's experiences. Unsolicited DPs were found to be sent for a variety of reasons, including to compliment the recipient and to coerce them into replying with sexual images. It is argued that MSM have trivialized unsolicited DPs and that these images are, according to current definitions, a form of technology-facilitated sexual violence that MSM experience on dating apps. However, there were variations in participants' experiences and some participants did not characterize unsolicited DPs as problematic or non-consensual, which challenges the notion that MSM always experience these images as sexually violent. These findings shed light on the complexities of unsolicited DPs and indicate the need to (re)examine definitions of technology-facilitated violence and explore MSM's consent practices within the context of dating apps.
\end{abstract}

Keywords Gay · MSM · Dating apps · Dick pics · Consent $\cdot$ Sexual violence

Christopher Dietzel

christopher.dietzel@dal.ca

1 School of Health and Human Performance, Dalhousie University, Halifax, Canada 


\section{Introduction}

Defined as a photo of a penis that is sent without verifying that the recipient wants to receive it, the unsolicited "dick pic" (DP) has been described as a common phenomenon in contemporary online dating culture (Mandau, 2019). Despite years of media attention on unsolicited DPs (Mandau, 2019; Paasonen et al., 2019), research in this field is limited and largely situates unsolicited DPs within the contexts of sexting and sexual harassment (Waling \& Pym, 2017; Waling et al., 2020). Literature on sexting generally frames unsolicited DPs as non-consensual, even though sexting itself can be consensual or non-consensual (Krieger, 2017). Within the literature on sexual harassment, DPs have been identified as a form of technology-facilitated sexual violence (TFSV), which is defined as a range of non-consensual sexual acts that are carried out online and in person (Powell \& Henry, 2017; Henry \& Powell, 2016).

Men who have sex with men (MSM) are concerned about experiencing TFSV when connecting with someone via online dating (Corriero \& Tong, 2016; Albury \& Byron, 2016; Bauermeister et al., 2014). These concerns are not unfounded, as recent research found that MSM experience sexual violence in both their online and in-person interactions with other dating app users (Dietzel, 2021). MSM dating app users are more than twice as likely as the general population to experience image-based sexual abuse (Waldman, 2019), and about one in three MSM has experienced unwanted sexual behavior online in the past 12 months (Jaffray, 2020). MSM's concerns about TFSV and the high rates of TFSV against them underscore the importance of understanding how MSM navigate issues of consent as they encounter unsolicited DPs on dating apps, particularly since about one in ten MSM feels disrespected or violated by such images (Marcotte et al., 2020).

Most of the research on TFSV and unsolicited DPs has focused on the experiences of heterosexual women, and few studies have investigated the experiences of MSM (Dietzel, accepted). Despite this, there are stark differences between women's and MSM's experiences. Women and are often targeted recipients of DPs, since heterosexual men send unsolicited DPs to coerce a recipient into engaging in a sexual activity (March \& Wagstaff, 2017), to pressure a recipient into sharing their own sexual images (Oswald et al., 2020), and to exert their power over a recipient and harm them (Vitis \& Gilmour, 2017). Heterosexual men often use unsolicited DPs as a vehicle for sexual harassment (Vitis \& Gilmour, 2017), and women mostly report negative responses to receiving them (Hayes \& Dragiewicz, 2018).

In contrast, MSM mostly report positive responses to receiving unsolicited DPs, describing them as arousing, flattering, and exciting (Marcotte et al., 2020; Tziallas, 2015). And unlike women who lack an equivalent to unsolicited DPs that they can use to fight back against sexual harassment (Oswald et al., 2020; Vitis \& Gilmour, 2017), MSM can reply to unsolicited DPs with images of their own (Waling \& Pym, 2017), thereby mitigating some of their power. Though men can send unsolicited DPs with the intention to harass, men also send them to get the recipient's attention (Paasonen et al., 2019), brag about their penis (Mandau, 
2019), express their sexual interest in the recipient (Oswald et al., 2020), and sexually arouse themselves and the recipient (Hayes \& Dragiewicz, 2018; Oswald et al., 2020).

\section{Power and Sexuality}

The differences in women's and MSM's experiences with unsolicited DPs are indicative of gender-based and sexuality-based power, and how men can exert their power through unsolicited DPs to perpetrate TFSV. However, with this power being more balanced between MSM, questions arise about the power that unsolicited DPs represent in MSM's socio-sexual interactions and how that power is exercised and experienced. Moreover, MSM can be both senders and recipients of unsolicited DPs, so their positionality as perpetrators and/or victims of TFSV is less fixed and more fluid. As such, it is unclear whether MSM perceive of themselves as perpetrators or victims of TFSV when sending and receiving unsolicited DPs.

The phenomenon of unsolicited DPs speaks to stereotypes about male sexuality and sexual consent. Men are perceived as always ready for sex and always eager to have sex (Snell et al., 1988). Similarly, according to Hollway's (1998) male sexual drive discourse, men are regarded as constantly pursuing sex and, as such, a man's consent is presumed. MSM deal with additional stereotypes because of their sexuality, like gay men being promiscuous (Klesse, 2016) and hypersexual (Anderson, 2010), which may further contribute to the perception that MSM always want sex and, in the case of unsolicited DPs, always want to receive such images.

\section{Sexual Consent}

It is important to distinguish between wanting to receive unsolicited DPs and consenting to receive unsolicited DPs. Consent is defined as a desire to engage in a sexual activity (Muehlenhard et al., 2016) and is not directly observable by other people (Hickman \& Muehlenhard, 1999; Muehlenhard et al., 2016). This means that wanting to engage in a sexual activity is distinctly different from consenting to engage in a sexual activity. People enact consent through agreement (Muehlenhard et al., 2016), thereby demonstrating their internal desire to engage (Hickman \& Muehlenhard, 1999). Consent can be communicated explicitly through written or verbal statements, or less explicitly through cues and signals (Muehlenhard et al., 2016).

Research shows that MSM prefer to be less explicit in their communications of consent, using non-verbal cues more often than verbal cues (Beres et al., 2004). There are also some MSM who consent to a sexual activity by not doing or saying anything to stop the advances of their partner (Beres et al., 2014). MSM's roles in seeking and securing consent are non-normative, making their sexual scripts less defined and more flexible (McKie, 2015; Klinkenberg \& Rose, 1994). However, these scripts may be unclear or open to interpretation, which can create challenges as MSM navigate issues of consent on dating apps, for example, feeling pressured to engage in sexual activities (McKie, 2015). And since unsolicited DPs are ubiquitous 
in contemporary online dating culture (Mandau, 2019), MSM may tacitly condone unsolicited DPs through unclear or misunderstood communications and behaviors.

Stereotypes about male sexuality (e.g., Snell et al., 1988) may also impact MSM's consent practices, as research has shown that MSM feel pressured to engage in sexual activities, even when they feel ambivalent or uninterested (McKie, 2015; Sweeney, 2014; Beres et al., 2014). However, it is important to recall that many MSM enjoy receiving unsolicited DPs (Marcotte et al., 2020; Tziallas, 2015) and that MSM engage in a variety of socio-sexual interactions that blur the lines between platonic and sexual (Byron et al., 2021; Race, 2015). In the case of unsolicited DPs, MSM's online interactions may complicate and obfuscate their consent practices, while also trivializing and perpetuating TFSV.

\section{Additional Complexities with Online Interactions}

Online affordances grant MSM unrestricted socio-sexual spaces where their interactions are liberated from traditional, heterosexual notions of sex and sexuality (Race, 2015). These affordances are shaped by the design and consumption of a product (Ingram et al., 2007) as well as users' behaviors, which influence the norms and culture of dating apps (Duguay et al., 2017).

Early research on MSM's online practices showed that MSM were intentional in their online interactions with other MSM, refraining from sharing too much too quickly out of concerns that the recipient would not share back (Jones, 2005). More recent research found that MSM who use dating apps experience tensions of openness-closedness and novelty-predictability as they consider what to share (or what not to share), when, and how (Fitzpatrick \& Birnholtz, 2018). These tensions may be reflected in MSM's experiences of sending and receiving unsolicited DPs since, for example, MSM may send such images to get the recipient's attention but recognize that the images are sent without the recipient's consent.

Lastly, MSM use dating apps for a variety of reasons, including for sexual and non-sexual reasons (Albury et al., 2019), and some MSM have expressed frustration with the prevalence of casual sexual interactions online (McKie et al., 2017). The variations in MSM's motivations for dating app use may mean that those who use apps for sexual purposes may welcome unsolicited DPs, while those who use apps for non-sexual purposes may experience them as TFSV.

\section{Methods}

The purpose of this study is to investigate MSM dating app users' experiences of sending and receiving unsolicited dick pics (DPs) and the factors that impact their experiences. I apply a consent framework to my analysis to explore how MSM understand and experience unsolicited DPs within the context of technology-facilitated sexual violence (TFSV).

This study is part of a research project that examined MSM dating app users' experiences with sexual violence. Data was collected in Fall 2017 from 25 
semi-structured interviews with self-identified MSM dating app users. Participants were recruited for 3 months using flyers posted at a large Canadian university and in the gay neighborhood of a large metropolitan Canadian city. Snowball sampling was also used to facilitate recruitment. Participants were required to be at least 18 years old and did not receive any compensation.

All participants identified as MSM who use dating apps. Twenty-four participants identified as men and one identified as gender-fluid. Twenty-two participants identified as gay, two as queer, and one as bisexual. None of the participants identified as transgender. Participants' ages ranged from 18 to 62, with about half of the participants younger than 25. More than half of the participants identified as white, though participants also identified as Middle Eastern, Hispanic, Multiracial, Asian, and Black. The names of the participants presented in this study are pseudonyms.

Grindr was the most used dating app among participants, though they also used other apps and websites, such as Tinder, Scruff, Hornet, and OkCupid. The average amount of time participants had been using dating apps was about 4 years, though it ranged from 1 month to 11 years. Participants spent an average of 7 hours a week on dating apps and their primary reasons for using the apps were looking for sex, meeting people, and pursuing dates or relationships.

Inspired by Blackwell et al. (2015), participants were offered three interview options (in person, telephone, online) to promote comfort, accessibility, and anonymity. Most participants chose to interview in person, though three were interviewed by phone and three via online chat. During the semi-structured interviews, I asked participants about their experiences of sending and receiving sexual images, including unsolicited DPs. I often posed follow-up questions to engage participants in personalized discussions about their experiences (Smith et al., 2009). I also asked participants about their understandings of consent and TFSV. The interview questions were developed following a review of studies on unsolicited DPs, consent, and TFSV, and they were designed to explore how MSM navigate consensual and nonconsensual sexual interactions. Each interview lasted about one hour and was conducted in either English or French. The audio from interviews was recorded and transcribed verbatim, and the text from online interviews was saved. The interview transcripts comprise the data analyzed in this study.

I analyzed the data using interpretive phenomenology, which explores how people make meaning of a phenomenon (Adams \& van Manen, 2008), to examine individual and collective MSM experiences and to attain a broader and deeper understanding of unsolicited DPs. In my analysis, I applied queer theory to scrutinize socio-cultural assumptions and critically examine power relations between MSM, particularly as they relate to consent negotiations and incidents of TFSV. Queer theory also allowed me to deconstruct normative ideologies and binaries (Gamson, 2000; Kirsch, 2000), notably, the perpetrator/victim (or perpetrator/survivor) binary often present in investigations of sexual violence. My analysis consisted of an iterative process of reading and re-reading the transcripts to identify passages related to unsolicited DPs. The passages were coded and organized according to themes (Braun \& Clarke, 2006). 


\section{Results}

Participants reported that MSM sent unsolicited DPs on dating apps without asking, and they used words like "undesired", "annoying", and "not respectful" to describe the receipt of such images. Participants also used words like "uncomfortable", "attacked", and "assaulted" to describe how they felt in those situations. Not all participants spoke negatively about unsolicited DPs, however. "Considering I'm there mostly for hooking up, that's totally okay with me. If they are opening up with a dick pic, it's great," said Jadyn (32, gay, male, Black). Other participants were unconcerned about receiving unsolicited DPs, including Mason (26, bisexual, male, Multiracial) who said, "If someone sends me a dick pic, most of the time I am indifferent."

Many participants identified unsolicited DPs as non-consensual. "There's no consent in the sense that they didn't ask you if you wanted to see their dick," said Hugo (23, queer, male, Hispanic). Similarly, Oscar (22, gay, male, White) said, "When people send photos straight away, I'm not consenting to receiving explicit photos." Other participants recognized the importance of seeking consent before sending unsolicited DPs, but hesitated to characterize them as non-consensual. Said Logan (18, gay, male, White), "I don't feel like I'm being assaulted, but I do feel like they should've asked." Mason was not sure that unsolicited DPs were "non-consensual", saying that he thought about them as "unwanted". Some participants, like Toby (20, gay, male, White) and Dion (37, gay, male, Middle Eastern), were undecided about the consensuality of unsolicited DPs. "I don't know if sending a dick pic is consensual or not," said Dion. "I actually don't know."

Many participants differentiated between consenting to receive unsolicited DPs and wanting to receive them. Elijah (18, gay, male, White) asserted that unsolicited DPs were non-consensual "by definition" but admitted that he sometimes wanted to receive them. Skyler (28, queer, male, White) thought of DPs as non-consensual, but said he was not "hugely bothered" by them. Robert (23, gay, male, White) expressed frustration in how society expected MSM to respond to unsolicited DPs:

For guys, it's weird saying no. It's almost that we cannot say no because, "Why would he not like it?" I think we have more pressure to accept it because we're guys... Everybody thinks that gay guys always want sex, so people feel more comfortable sending pictures because they think everybody is going to like it, especially if it's a nice dick.

Other participants felt indifferent about unsolicited DPs. "I knew I would be getting dick pics on Grindr, so I wasn't terribly uncomfortable with it," said William (25, gay, male, White). Hugo agreed: "It has become normalized. Even if you don't want it, you're just like 'whatever'."

Participants spoke about unsolicited DPs as a standard part of their dating app experience, and several participants reported receiving them regularly. For example, Michael (18, gay, male, White) said, "If I check my messages every couple of days, I will get at least one, usually more." Despite this, some participants emphasized that they did not like to receive unsolicited DPs. "I think if they were up to me, they 
wouldn't happen. But some people obviously respond positively because, if they didn't, I don't think people would do it at all," said Michael. Skyler asserted that unsolicited DPs were "only semi-normalized" because some MSM enjoy receiving them, while others do not. Oscar agreed: "I don't see that as normal, but a lot of people-everyone on the app-feel that it's for that specific purpose."

Receiving unsolicited DPs was not a common occurrence for all participants. William said that "not too many people" had sent him unsolicited DPs in the year and a half that he used Grindr. Ang (36, gay, male, Asian) reported only receiving one unsolicited DP in his ten years on the apps and said it was "because I don't fit the gay male definition of beauty-I am not a white cisgender male." Robert did not specify how many he had received, but he expressed frustration with the uncertainty of not knowing when he would receive one: "You never know when you receive a message if it's going to be someone being nice or just a random person sending you a nude picture."

Some participants stated that unsolicited DPs reflected the sexual nature of MSM dating apps. "Grindr allows people to meet up and have hook-ups, which I think is part of the point. But then, within that same platform, it also allows for these non-consensual acts," said William. Other participants noted that the apps' names implied that users would receive sexual images. "It's called Grindr, so I expect it to be full of horny people... it's [receiving unsolicited DPs] not something that shocks me, for that reason," explained Hugo. A few participants felt that being logged into a dating app signaled an openness to receiving sexual advances from other users. For example, Ravi (28, gay, male, Middle Eastern) asserted that the sexual nature of the app-space contributed to the assumption that users want to receive unsolicited DPs: "The culture of the application is giving the idea that we consent already."

Joe's (31, gay, male, White) perspective on unsolicited DPs changed during his interview. At first, he said that MSM should be open to receiving such images because they consent to using dating apps, which are known to be highly sexual. "If you're a member of the site, then [consent] is implied," he stated. Later in the interview, however, Joe compared sending and receiving unsolicited DPs to the paradox of the chicken and the egg:

What comes first? I'm making it so different steps come first. I'm saying that if you are a member of Grindr, then you need to consent to dick pics, because people send dick pics. But what if we swapped that and say, if you are a member of Grindr then you cannot send dick pics, because people do not consent to them? If many people using this app don't want unsolicited dick pics, then who's to say this should be a normalized behavior?

Several participants tried to make sense of unsolicited DPs by comparing them to in-person examples. "If you flash someone on the street, that's non-consensual and its illegal. On Grindr, its non-consensual but it just makes you roll your eyes," explained Logan. Similarly, Joe said, "I don't mind being sent an unsolicited dick pic in the same way I would mind being flashed in public. What behaviors we consent to change whether it's in virtual or 3D space." Jadyn asserted that the affordances of the internet allow MSM to comport themselves differently on dating apps than in person: "Obviously, someone does not walk up to you in a bar, pull down 
their pants, and show you their dick. People say or do things online that in real life you would never do." Logan agreed: "Unsolicited nudes—-that just doesn't happen in real life, but on Grindr it happens so much that it's normal."

\section{Factors that Affected Participants' Experiences}

Participants identified factors that had an impact on how they felt receiving unsolicited DPs, such as the timing of when the images were received. Noah (28, gay male, White) found unsolicited DPs "too direct" and "imposing" when he received them right away, saying that he appreciated "having a short discussion" first. Similarly, William said there was "a certain amount of engagement that needs to happen" before he felt comfortable receiving a DP: "Even if your intention is only to hook up, you want to ease into a conversation." Mason agreed: "The earlier the dick pic comes, the less I'm willing to see it." Toby, however, thought it was "ridiculous" that MSM would feel more comfortable receiving an unsolicited DP after exchanging a few messages. "What difference does three different lines of convo make, between sending a dick pic or whatever?" asked Toby.

For some participants, the longer they used dating apps, the more banal unsolicited DPs became. "I'm really unmoved now. I used to be really shocked when I was at the beginning, in my early stages of usage in the apps," said Theo (20, gay, gender-fluid, Multiracial). Ravi found unsolicited DPs less arousing after being on the apps for 6 years: "I'm not excited to see them anymore because I have seen so many." Dion, who had been using the apps for 4 years, agreed: "I have become kind of become immune or numb to it." Although Logan had become more comfortable sending and receiving sexual images after spending a year on the apps, he felt conflicted about getting used to receiving unsolicited DPs: "It's kind of fucked up that it doesn't bother me. Someone is sexually exposing themselves without me asking, but it sort of just seems normal now in the context."

Some participants reported that their mood impacted how they felt receiving unsolicited DPs. Said Hugo, "If I'm in a lazy mood, I'll probably make a joke about it or be like, 'Nice try.' If I'm busy, I will probably block the profile." For Oscar, the word "mood" was a euphemism for sexual arousal such that the more "in the mood" he was, the more willing he was to receive an unsolicited DP. Both Elijah and Gabriel (21, gay, male, White) said that if they were "horny", they would be happy to receive unsolicited DPs.

Several participants reported that attractiveness made a difference in how they felt receiving unsolicited DPs. Dion said it could either be a "good outcome" or "bad outcome" depending on the sender and the DP: "If it's a photo that I find nice, it could be positive. But if it's a photo that I think is not nice, it would be a negative interaction." Charlie (24, gay, male, White) also said that his reaction depended on attractiveness: "If it's nice, sometimes I'll compliment them." Other participants talked about ignoring the sender's advances, deleting the chat, or blocking the user-unless they found the person or the DP attractive. "I cut the conversation clear, unless the person is so hot that I can't resist," explained Ravi. Similarly, Toby said, "If I haven't spoken to them and they send me a dick pic, I don't feel inclined 
to respond-unless I'm attracted to it." Elijah recognized that the attractiveness of the DP affected his reaction: "I'm not proud of it, I know that it's contradictory, but that's honestly the way it is for me."

Participants explained that their reasons for using dating apps also contributed to their experiences. Ravi emphasized that he did not like receiving unsolicited DPs if he was "chilling on a Thursday night and having a glass of wine and just looking to chat with people." Jadyn reported having different reactions when he was looking for casual sex compared to when he was looking for a relationship: "If I am in dating mode and have a cute picture and am looking to date, getting random unsolicited dick pics, that can be viewed as harassment."

\section{Reasons for Sending Unsolicited Dick Pics}

Participants gave a few reasons for why MSM send unsolicited DPs. "Often people start a conversation with nudes to get your attention," said Toby. Michael agreed: "They think it'll get people's attention because nude imagery is attention grabbing." Participants also said unsolicited DPs could be used to assess another person's interest. "When someone is sending you a picture, he's trying to ask you, 'Are you attracted to me?"' said Nicolas (62, gay, male, Multiracial). Similarly, Jadyn explained that sending unsolicited DPs helped MSM gauge another user's willingness to chat: "In this world of applications, we're trying to message each other and find out if people are interested."

Participants said that unsolicited DPs could be used to inform the receipt about the sender's physical attributes. "The reason I share is because I would like to see what I'm going to end up with. It's more about being open and transparent and honest," said Karam (46, gay, male, Middle Eastern). Ravi found unsolicited DPs informative: "It's just to show the person that this is you. This is how you look like from the side, from the angles, from the back."

Several participants identified sexual arousal as a reason why MSM dating app users send unsolicited DPs. Said Elijah, "I'm really horny, sometimes I'll send one of those." Charlie talked about friends of his who were exhibitionists and enjoyed sending unsolicited DPs for their own pleasure. Joe said that he would send unsolicited DPs when he was looking for sex, but that it was not something he would do regularly: "It's only when I've reached a certain point of how long it's been since I've last been with a man."

Participants asserted that time of day, drug and alcohol consumption, and feelings of loneliness also factored into MSM's decisions of whether to send an unsolicited DP. "At 3 AM when everybody's drunk, that's when the most dick pics happen. But at 1 PM, nobody is going to send me a dick pic. Usually, it's later in the evening when people are lonely," said Robert. Dion explained that MSM who use drugs during sex-a practice referred to a "PnP" (Party and Play)—send unsolicited DPs because they are "high and very horny and looking for lots of sex."

A few participants said that MSM send unsolicited DPs to persuade the recipient into engaging in a sexual activity. "I think people send it out because they want to entice you to sleep with them," said Robert. Nicolas explained: 
They'll send you a picture of their penis to brag on how well-endowed they are. And usually, it has to do with what they feel will entice you. Because, don't forget, they are approaching me, and they want to convince me. They are trying to advertise... they're trying to put their best foot forward to entice me into meeting with them.

Participants also said that some MSM would send unsolicited DPs to coax other users into replying with DPs of their own. "They send one and they always expect one in return," said Robert. As such, some participants reported feeling pressured or obligated to reply with sexual images, even when they were uninterested. "Sometimes if they send me their photos first, even if I don't want it to continue, I'll send mine back as a tit-for-tat," said Joe.

Some participants found it challenging to gauge whether sending unsolicited DPs would help or hurt their chances of connecting with other MSM online. Michael talked about going through a process of "trial and error" to determine an "effective strategy" for engaging other users. Pierre (25, gay, male, White) said, "It's difficult to know when to be direct or not direct. There's a tension between the two." William asserted that MSM who were new to dating apps might imitate other users' behaviors instead of doing what makes them comfortable: "If you don't have as much experience with your own and other people's sexuality, then you might base yourself more off of social scripts and because of that, you might have a greater tendency to go towards normalized behaviors."

\section{Discussion}

An analysis of participants' experiences revealed three "dimensions" of unsolicited DPs: consensual, wanted, and typical. The consensual dimension denotes the extent to which an MSM app user consents to receiving unsolicited DPs. The wanted dimension describes the extent to which an MSM dating app user desires, and is willing to receive, unsolicited DPs. The typical dimension refers to the extent to which it is standard and common for an MSM dating app user to receive unsolicited DPs. These three dimensions, each of which exists on a continuum, capture MSM dating app users' experiences of receiving unsolicited DPs.

These three dimensions intersect such that MSM can simultaneously experience unsolicited DPs in terms of being consensual, wanted, and/or typical (see Fig. 1). MSM's experiences within and across the three dimensions are not fixed, and their experiences can shift on each continuum according to internal and external factors as well as interactions with different dating app users. These three dimensions capture a range of MSM dating app users' experiences and build on previous research, which had characterized unsolicited DPs in terms of desire, expectations, and harassment (Amundsen, 2020; Mandau, 2019; Paasonen et al., 2019; Waling \& Pym, 2017). Findings from the present study highlight the diversity and range of MSM's experiences with unsolicited DPs and demonstrate that MSM have complex - and sometimes conflicting or contradictory-reactions to receiving unsolicited DPs. 


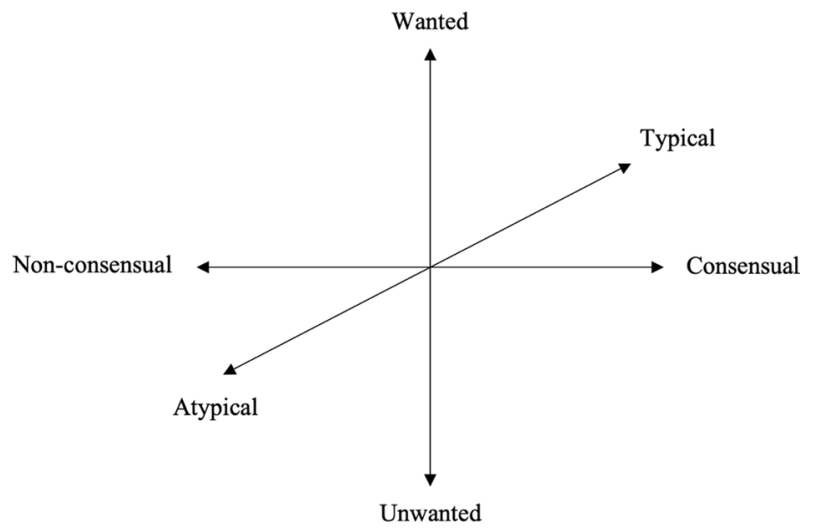

Fig. 1 The three dimensions of unsolicited dick pics

Many participants characterized the receipt of unsolicited DPs as harmful and non-consensual, which reflects the language used to describe unwanted sexual advances, online sexual harassment, and image-based sexual abuse (Henry \& Powell, 2018). As such, findings show that MSM experience unsolicited DPs as a form of TFSV, as the term is currently conceptualized (Powell \& Henry, 2017).

However, several participants were unsure or undecided about the extent to which sending and receiving unsolicited DPs is non-consensual. Other participants described wanting to receive such images and actively welcomed them from other MSM dating app users. These findings suggest that not all MSM perceive unsolicited DPs as problematic, non-consensual, or sexually violent. Furthermore, participants explained that their experiences of receiving unsolicited DPs could shift from being less consensual to more consensual depending on the sender, the DP, and other factors. This finding challenges the assumption that unsolicited DPs are always a form of TFSV and raises questions about whether the concept of TFSV, which has been developed from understandings of sexual interactions between heterosexual men and women, appropriately captures the realites of MSM's socio-sexual relationships. This finding also raises questions about how, as Passonen et al. (2019) noted, elements such as identity, power, and sociability can affect people's interpretations of and experiences with unsolicited DPs.

Participants explained that the culture of MSM dating apps implies that all MSM enjoy receiving unsolicited DPs and they asserted that MSM who express discomfort or disinterest in unsolicited DPs would be viewed negatively. This finding reflects stereotypes about male sexuality, including the assumption that men are constantly pursuing sex, are always eager to have sex, and consent to any sexual advance (Hollway, 1998; Snell et al., 1988). MSM who subscribe to these assumptions may send unsolicited DPs without hesitation or concern because they expect other MSM to be interested in and consent to their sexual advances. Similarly, as participants explained, MSM may feel pressured to respond positively to unsolicited DPs and conform to MSM dating app culture. This suggests that MSM may hesitate to challenge non-consensual sexual advances like unsolicited DPs because of the 
assumptions that all MSM send unsolicited DPs and that all MSM enjoy receiving them.

Additionally, data analysis revealed that MSM may dismiss their own feelings about unsolicited DPs as a form of TFSV because of assumed expectations about what MSM should want or should enjoy. This is troubling but not surprising, as other research has shown that MSM minimize incidents of sexual violence and do not see themselves as victims of sexual violence (Braun et al., 2009; Davies, 2002). And since the receipt of unsolicited DPs may not immediately or visibly impact their health or safety, MSM may disregard such images and dismiss feelings of discomfort as insignificant or unimportant. However, this dismissal feeds into the trivialization of TFSV on dating apps and perpetuates the perception that MSM want to receive and consent to receiving unsolicited DPs.

Many participants expected to receive unsolicited DPs on MSM dating apps, and they used words like "normalized" to describe the regularity of this practice. This is not surprising since unsolicited DPs have been framed as a standard part of online dating (Mandau, 2019) and MSM dating apps have a reputation for being highly sexual spaces (Race, 2015). Together, this suggests that sending unsolicited DPs is a "social norm" (Ross et al., 1977) in MSM dating app culture-a norm that app companies like Grindr recently recognized. ${ }^{1}$ That said, participants noted a tension between the assumption that MSM dating app users send and receive unsolicited DPs and the reality that not all MSM dating app users have sent or received such images. Thus, the question arises as to whether sending and receiving unsolicited DPs is a perceived norm or an actual norm (Ross et al., 1977) in MSM dating app culture.

Findings from this study suggest that sending and receiving unsolicited DPs is a perceived norm in MSM dating app culture since not all participants sent unsolicited DPs and some participants rarely received any. Findings also reveal that, contrary to how unsolicited DPs have been framed in popular culture and previous studies (e.g., Vitis \& Gilmour, 2017), not all MSM send unsolicited DPs and the frequency and quantity of unsolicited DPs received by MSM varies.

Similarly, several participants pushed back against the notion that sending unsolicited DPs is "normalized" in MSM dating app culture, with one participant saying that the practice is only "semi-normalized" because not all MSM enjoy receiving them. In challenging the normalcy of this phenomenon, participants differentiated between how they felt receiving unsolicited DPs and how they thought other MSM felt. These participants minimized or excused their feelings because they had the impression that unsolicited DPs were a typical (and wanted) practice among MSM, which speaks to stereotypes about male sexuality, notably, that men are always ready for sex and always pursuing sex (Hollway, 1998; Snell et al., 1988). As findings from this study show, these stereotypes fuel assumptions about MSM's interests in receiving unsolicited DPs and perpetuate a culture of TFSV on dating apps.

\footnotetext{
1 In Summer 2019, Grindr updated its software to include an option for users to indicate their willingness to receive "NSFW Pics" on their profile (Dietzel, 2021; Grindr, n.d.).
} 


\section{Seven Factors that Impact the Three Dimensions}

Data analysis revealed several factors that can affect MSM dating app users' experiences of receiving unsolicited DPs: (1) the timing of when in a chat the unsolicited DP is received; (2) the amount of time the recipient has been using dating apps; (3) the mood of the recipient, including their level of sexual arousal; (4) the recipient's reasons for using MSM dating apps, such as looking for sex or dates; and (5) the attractiveness of the person sending the unsolicited DP and/or the attractiveness of the DP itself. Participants also stated that time of day, drug and alcohol consumption, and feelings of loneliness had an impact on MSM's motivations. Since feelings of loneliness can be categorized under mood, there are two additional factors that can affect MSM's experiences: (6) the time of day when the unsolicited DP is received; and (7) the consumption of drugs and/or alcohol. These findings align with previous research, which had suggested that MSM may respond differently to unwanted sexual images depending on how long they had been using MSM dating apps (Pingel et al., 2013), the attractiveness of dating app users and their photos (Chan, 2017), the time of day and timing in a conversation (Albury \& Byron, 2016), and the consumption of drugs and alcohol (Benotsch et al., 2013; Dake et al., 2012; Yeung et al., 2014).

Though more research needs to be done to understand the extent to which these seven factors impact how MSM respond to unsolicited DPs, the fact that MSM's experiences can shift according to these factors shows that their experiences exist on a continuum. Additionally, this finding challenges the idea that MSM who receive unsolicited DPs are automatically victims of TFSV, since these factors can transform a non-consensual experience into a (more) consensual one.

Of the seven factors, there are four that hold particular implications within the contexts of consent and TFSV. Below, I discuss how timing in a chat, time spent using MSM dating apps, sexual arousal, and attractiveness relate to issues of consent and TFSV among MSM.

\section{Timing in a Chat}

Some participants explained that when they received unsolicited DPs in their inapp chats made a difference to their experience, such that the sooner the images arrived the less wanted and/or consensual they were, and the later they arrived the more wanted and/or consensual they were. Research on consent has found that some people want to establish mutual trust and understanding before engaging in a sexual activity (Barker, 2013; Bauer, 2014), and that sexual interactions are perceived as more consensual the longer the relationship (Humphreys, 2007; Humphreys \& Herold, 2007). Together, this suggests that MSM may experience unsolicited DPs as more wanted and/or consensual after some form of interaction with the sender because they feel that they have developed a level of trust and understanding with that person, perhaps even a basic relationship. 


\section{Time Spent Using MSM Dating Apps}

The amount of time that participants had used dating apps impacted their experiences of receiving unsolicited DPs. Since MSM learn sexual scripts from other MSM that they interact with online (Simon \& Gagnon, 2003), they may understand unsolicited DPs as part of a sexual script and, consequently, learn that sending unsolicited DPs is an acceptable way to interact. This suggests that the more experience MSM have with dating apps, the more they grow accustomed to sexual scripts that embed unsolicited DPs in the culture of MSM dating apps. This process fuels a cycle in which app norms are reinforced by their users (Ingram et al., 2007; Duguay et al., 2017), resulting in an app culture that trivializes and perpetuates TFSV.

\section{Sexual Arousal}

Participants reported that their level of sexual arousal made a difference in their experiences of receiving unsolicited DPs. Since many MSM use dating apps for sexual purposes (Albury et al., 2019), receiving sexual advances from other MSM may be welcomed. Additionally, as Tziallas (2015) noted, it can be exciting for MSM to receive sexual images from other users on dating apps since the images represent possibility. Dating apps connect users within close geographic proximity, which means that when an MSM dating app user receives an unsolicited DP, the sender is physically located near the recipient. For MSM who are on dating apps in pursuit of sexual activity, the receipt of an unsolicited DP could signal the potential to meet someone for an in-person sexual encounter-and perhaps soon (Licoppe et al., 2016). As such, MSM who receive unsolicited DPs may minimize concerns about consent or forgo conversations about consent when presented with the prospect of an immediate sexual interaction.

\section{Attractiveness}

The attractiveness of the person who sent an unsolicited DP made a difference in participants' experiences, as did the attractiveness of the DP itself. This is not surprising as research has shown that MSM prioritize a man's physical appearance (Kozak et al., 2009) and objectify other MSM on dating apps (Anderson et al., 2018; Tziallas, 2015). However, it is important to note that the attractiveness of perpetrators of sexual harassment can influence how they are perceived, such that a male perpetrator who is more stereotypically attractive is perceived as less harassing (LaRocca \& Kromrey, 1999). Thus, MSM may disregard the unwanted or non-consensual nature of unsolicited DPs in favor of a sexual interaction with an attractive person or an attractive penis.

\section{Sending Unsolicited Dick Pics}

Participants reported a variety of motivations that MSM have for sending unsolicited DPs and many of them align with previous research, including to get the 
recipient's attention (Paasonen et al., 2019), brag and compliment the recipient (Mandau, 2019), sexually arouse themselves (Hayes \& Dragiewicz, 2018; Oswald et al., 2020), and solicit sexual images from the recipient (Oswald et al., 2020). Notably, participants reported that MSM send unsolicited DPs to coax the recipient into replying with DPs of their own. Online sexual coercion has been identified as a form of TFSV, along with other behaviors such as imaged-based sexual abuse and sexual harassment (Powell \& Henry, 2017; Henry \& Powell, 2016). Thus, sending unsolicited DPs appears to be a tactic that MSM employ to coerce other dating app users into engaging in a sexual activity with them, thereby perpetrating TFSV against other MSM.

However, the reputation and culture of MSM dating apps as sexual spaces (Race, 2015) coupled with the diversity of user expectations and goals (Albury et al., 2019), creates a situation in which users may have difficulty in deciphering who is online for what and, consequently, what is perceived or experienced as TFSV. For MSM who use dating apps for sexual purposes, an unsolicited DP may be welcome and sexually arousing. For MSM who use dating apps for other reasons, an unsolicited DP may be unwelcome, harassing, or non-consensual.

Additionally, since participants explained that sending unsolicited DPs is a way for MSM to get the recipient's attention and gauge their interest, these images may be intended as a conversation starter. Unsolicited DP may be an online version of "peacocking" (Strauss, 2005)—flaunting one's assets to a potential partner, communicating (sexual) desire, and asking them to return the gesture if they are interested. This does not diminish the fact that MSM may experience unsolicited DPs as nonconsensual; rather, this suggests that MSM have trivialized this sexual advance and reframed it as an invitation for the recipient to engage with the sender.

\section{Conclusion}

In the past few years, there has been a renewed attention to issues of consent and TFSV, which has been fueled by the \#MeToo movement and high rates of sexual and domestic violence during the lockdown periods of the COVID-19 pandemic (Dietzel $\&$ Shariff, accepted). This has prompted governments to implement revised sex education curricula that teach students about TFSV (e.g., Government of Quebec, 2021) and prevention programs that address sexual violence against LGBTQ+ people (e.g., Ending Violence Association of British Columbia, n.d.). Many Canadian provinces have also enacted legal mandates that require universities to have stand-alone policies against sexual violence (Busby, accepted; Crocker \& Dufour, accepted). Dating app companies have shared resources about sexual violence and consent on their websites, like Tinder's "Dating Safety Tips" (Tinder, n.d.). These app companies have also implemented new software to encourage safe, healthy, and consensual interactions among their users, including Grindr, which now offers an option for its users to indicate on their profile the extent to which they are open to receiving "NSFW Pics" (Grindr, n.d.). Community organizations have launched campaigns to raise awareness about TFSV, including the \#ÇaExiste campaign in Quebec (RÉZO, 2021). Other community organizations, like Hollaback!, have developed free 
information and resources, such as a digital safety kit to help people address online harassment (Hollaback!, 2021). These various initiatives highlight some of the many ways that institutions and organizations have been working to address TFSV and encourage safe, consensual interactions online. As such, findings from this study can be applied to help develop policies, resources, software, and campaigns that address TFSV among MSM.

For many MSM dating app users, unsolicited DPs are non-consensual sexual advances that are experienced as TFSV. However, not all MSM dating app users perceive unsolicited DPs to be problematic, harmful, or sexually violent. While MSM may recognize unsolicited DPs as non-consensual and/or a form of TFSV, this study showed that MSM dating app users have minimized and condoned unsolicited DPs. Unsolicited DPs are embedded in the sexual scripts and norms of MSM dating app culture, practices that MSM dating app users learn and internalize over time and, subsequently, perpetuate in their interactions with other MSM. The phenomenon of unsolicited DPs is entrenched within the culture of MSM dating apps and MSM are often complicit in maintaining beliefs, practices, and systems that trivialize and sustain TFSV against MSM on dating apps.

This study revealed that MSM's experiences with unsolicited DPs range across the dimensions of consensual, wanted, and typical and can be impacted by several factors, including attractiveness and sexual arousal. Findings therefore indicate that unsolicited DPs are a more complex phenomenon that may not be fully understood within current consent and TFSV frameworks.

There are limitations to this research. Since most participants identified as young, white, and cisgender, a more diverse sample of MSM dating app users would allow for a more in-depth investigation of how identity can impact MSM's experiences with unsolicited DPs. Additionally, given the qualitative nature of this research, a quantitative project could investigate relationships between the three dimensions and the seven factors identified in this study. Such research could also explore whether other internal and external factors impact MSM's experiences of receiving unsolicited DPs. Lastly, recognizing the uniqueness of the phenomenon of unsolicited DPs, this study's findings about consent and TFSV may not apply to other online sexual interactions among MSM dating app users. There is an urgent need to investigate the consent practices of MSM dating app users and how their online interactions may impact their safety and health. Future studies should queer the concept of TFSV through theoretical and empirical research to ensure it accurately captures and applies to MSM's experiences, including their experiences with dating apps.

Acknowledgements The author would like to thank Dr. Ada Sinacore for her contributions to this paper.

Authors' Contributions Christopher Dietzel is the sole author of this paper.

Funding This research was supported by the Fonds de Recherche du Québec - Société et Culture (FRQSC) Grant 2019-B2Z-259244 and by iMPACTS: Collaborations to Address Sexual Violence on Campus; Social Sciences and Humanities Research Council of Canada (SSHRC) Partnership Grant 8952016-1026 (Project Director, Shaheen Shariff, Ph.D., James McGill Professor, McGill University). 


\section{Declarations}

Conflicts of Interest The author has none to report.

Data Availability The data from this study were collected by the author in Fall 2017. The author received ethics approval from the Research Ethics Board of McGill University.

\section{References}

Adams, C., \& van Manen, M. (2008). Phenomenology. In L. M. Given (Ed.), The SAGE encyclopedia of qualitative research methods (pp. 614-619). SAGE Publications.

Albury, K., Byron, P., McCosker, A., Pym, T., Walshe, J., Race, K., et al. (2019). Safety, risk and wellbeing on dating apps: Final report. Swinburne University of Technology.

Albury, K., \& Byron, P. (2016). Safe on my phone? Same-sex attracted young people's negotiations of intimacy, visibility, and risk on digital hook-up apps. Social Media + Society, 2(4), 1-10.

Amundsen, R. (2020). 'A male dominance kind of vibe': Approaching unsolicited dick pics as sexism. New Media \& Society, 23(6), 1465-2148.

Anderson, J. R., Holland, E., Koc, Y., \& Haslam, N. (2018). iObjectify: Self-and other-objectification on Grindr, a geosocial networking application designed for men who have sex with men. European Journal of Social Psychology, 48(5), 600-613.

Anderson, K. J. (2010). Benign bigotry: The psychology of subtle prejudice. Cambridge University Press.

Barker, M. (2013). Consent is a grey area? A comparison of understandings of consent in Fifty Shades of Grey and on the BDSM blogosphere. Sexualities, 16(8), 896-914.

Bauer, R. (2014). Negotiating critical consent. Queer BDSM Intimacies (pp. 75-106). Palgrave Macmillan.

Bauermeister, J. A., Yeagley, E., Meanley, S., \& Pingel, E. S. (2014). Sexting among young men who have sex with men: Results from a national survey. Journal of Adolescent Health, 54, 606-611.

Benotsch, E. G., Snipes, D. J., Martin, A. M., \& Bull, S. S. (2013). Sexting, substance use, and sexual risk behavior in young adults. Journal of Adolescent Health, 52(3), 307-313.

Beres, M. A., Herold, E., \& Maitland, S. B. (2004). Sexual consent behaviors in same-sex relationships. Archives of Sexual Behavior, 33(5), 475-486.

Beres, M. A., Senn, C. Y., \& McCaw, J. (2014). Navigating ambivalence: How heterosexual young adults make sense of desire differences. Journal of Sex Research, 51(7), 765-776.

Blackwell, C., Birnholtz, J., \& Abbott, C. (2015). Seeing and being seen: Co-situation and impression formation using Grindr, a location-aware gay dating app. New Media \& Society, 17(7), 1117-1136.

Braun, V., \& Clarke, V. (2006). Using thematic analysis in psychology. Qualitative Research in Psychology, 3(2), 77-101.

Braun, V., Schmidt, J., Gavey, N., \& Fenaughty, J. (2009). Sexual coercion among gay and bisexual men in Aotearoa/New Zealand. Journal of Homosexuality, 56(3), 336-360.

Busby, K. (accepted). Eliminating adversarial hearings from sexual violence complaint processes. In S. Shariff \& C. Dietzel (Eds.), IMPACTS: Reclaiming the role of universities to address sexual violence through multi-sector partnerships in law, arts and social media. University of Toronto Press.

Byron, P., Albury, K., \& Pym, T. (2021). Hooking up with friends: LGBTQ+ young people, dating apps, friendship and safety. Media, Culture \& Society, 43(3), 497-514.

Chan, L. S. (2017). Ambivalence in networked intimacy: Observations from gay men using mobile dating apps. New Media \& Society, 20(7), 2566-2581.

Corriero, E. F., \& Tong, S. T. (2016). Managing uncertainty in mobile dating applications: Goals, concerns of use, and information seeking in Grindr. Mobile Media \& Communication, 4(1), 121-141.

Crocker, D., \& Dufour, G. (accepted). Developing campus anti-violence policies: Lessons learned from the criminalization of gender-based violence. In S. Shariff \& C. Dietzel (Eds.), IMPACTS: Reclaiming the role of universities to address sexual violence through multi-sector partnerships in law, arts and social media. University of Toronto Press.

Dake, J. A., Price, J. H., Maziarz, L., \& Ward, B. (2012). Prevalence and correlates of sexting behavior in adolescents. American Journal of Sexuality Education, 7(1), 1-15. 
Davies, M. (2002). Male sexual assault victims: A selective review of the literature and implications for support services. Aggression and Violent Behavior, 7(3), 203-214.

Dietzel, C. (accepted). Exploring connections between sexual violence and dating apps: A focus on the experiences of men who have sex with men. In C. Dietzel \& S. Shariff (Eds.), IMPACTS: Reclaiming the role of universities to address sexual violence through multi-sector partnerships in law, arts, and social media. University of Toronto Press.

Dietzel, C. (2021). "That's straight-up rape culture": Manifestations of rape culture on Grindr. In J. Bailey, A. Flynn \& N. Henry (Eds.), The Emerald international handbook of technology-facilitated violence and abuse (pp. 351-368). Emerald.

Duguay, S. C., Burgess, J., \& Light, B. (2017). Mobile dating and hookup app culture. In P. Messaris \& L. Humphreys (Eds.), Digital media: Transformations in human communication (2nd ed., pp. 213-221). Peter Lang.

Ending Violence Association of British Columbia (n.d.). Western Canada Sexual Assault Initiative. https://endingviolence.org/prevention-programs/western-canada-sexual-assault-initiative/.

Fitzpatrick, C., \& Birnholtz, J. (2018). "I shut the door": Interactions, tensions, and negotiations from a location-based social app. New Media \& Society, 20(7), 2469-2488.

Gamson, J. (2000). Sexualities, queer theory, and qualitative research. In N. K. Denzin \& Y. S. Lincoln (Eds.), Handbook of qualitative research (2nd ed., pp. 347-365). Sage.

Government of Quebec (2021). Sexuality education. http://www.education.gouv.qc.ca/en/teachers/dossi ers/sexuality-education/.

Grindr (n.d.). Building your profile. https://help.grindr.com/hc/en-us/articles/4402336949523-Buildingyour-profile.

Hayes, R. M., \& Dragiewicz, M. (2018). Unsolicited dick pics: Erotica, exhibitionism or entitlement? Women's Studies International Forum, 71, 114-120.

Henry, N., \& Powell, A. (2018). Technology-facilitated sexual violence: A literature review of empirical research. Trauma, Violence, \& Abuse, 19(2), 195-208.

Henry, N., \& Powell, A. (2016). Sexual violence in the digital age. Social \& Legal Studies, 25, 397-418.

Hickman, S. E., \& Muehlenhard, C. L. (1999). "By the semi-mystical appearance of a condom": How young women and men communicate sexual consent in heterosexual situations. Journal of Sex Research, 36(3), 258-272.

Hollaback! (2021). HeartMob: Online harassment resources. https://iheartmob.org/resources.

Hollway, W. (1998). Gender difference and the production of subjectivity. In J. Henriques, W. Hollway, C. Urwin, C. Venn, \& V. Walkerdine (Eds.), Changing the subject: Psychology, social regulation and subjectivity (pp. 223-261). Methuen.

Humphreys, T. (2007). Perceptions of sexual consent: The impact of relationship history and gender. Journal of Sex Research, 44(4), 307-315.

Humphreys, T., \& Herold, E. (2007). Sexual consent in heterosexual relationships: Development of a new measure. Sex Roles, 57, 305-315.

Ingram, J., Shove, E., \& Watson, M. (2007). Products and practices: Selected concepts from science and technology studies and from social theories of consumption and practice. Design Issues, 23(2), $3-16$.

Jaffray, B. (2020). Experiences of violent victimization and unwanted sexual behaviours among gay, lesbian, bisexual and other sexual minority people, and the transgender population, in Canada, 2018. Statistics Canada. https://www150.statcan.gc.ca/n1/pub/85-002-x/2020001/article/00009-eng.htm.

Jones, R. H. (2005). 'You show me yours, I'll show you mine': The negotiation of shifts from textual to visual modes in computer-mediated interaction among gay men. Visual Communication, 4(1), 69-92.

Kirsch, M. H. (2000). Queer theory and social change. Routledge.

Klesse, C. (2016). The spectre of promiscuity: Gay male and bisexual non-monogamies and polyamories. Routledge.

Klinkenberg, D., \& Rose, S. (1994). Dating scripts of gay men and lesbians. Journal of Homosexuality, 26, 23-35.

Kozak, M., Frankenhauser, H., \& Roberts, T.-A. (2009). Ob- jects of desire: Objectification as a function of male sexual orientation. Psychology of Men \& Masculinity, 10(3), 225-230.

Krieger, M. A. (2017). Unpacking "sexting": A systematic review of nonconsensual sexting in legal, educational, and psychological literatures. Trauma, Violence, \& Abuse, 18(5), 593-601.

LaRocca, M. A., \& Kromrey, J. D. (1999). The perception of sexual harassment in higher education: Impact of gender and attractiveness. Sex Roles, 40(11), 921-940. 
Licoppe, C., Rivière, C. A., \& Morel, J. (2016). Grindr casual hook-ups as interactional achievements. New Media \& Society, 18(11), 2540-2558.

Mandau, M. B. H. (2019). 'Directly in Your Face': A qualitative study on the sending and receiving of unsolicited 'dick pics' among young adults. Sexuality \& Culture, 24(1), 72-93.

March, E., \& Wagstaff, D. L. (2017). Sending nudes: Sex, self-rated mate value, and trait Machiavellianism predict sending unsolicited explicit images. Frontiers in Psychology, 8, 1-6.

Marcotte, A. S., Gesselman, A. N., Fisher, H. E., \& Garcia, J. R. (2020). Women's and men's reactions to receiving unsolicited genital images from men. The Journal of Sex Research, 58, 412.

McKie, R. M. (2015). "Just say yes" - Sexual consent and boundary setting on- and offline: An international perspective of men of varying sexual orientations (Master's thesis). Retrieved from Trent University Graduate Thesis Collection.

Muehlenhard, C. L., Humphreys, T. P., Jozkowski, K. N., \& Peterson, Z. D. (2016). The complexities of sexual consent among college students: A conceptual and empirical review. The Journal of Sex Research, 53(4-5), 457-487.

Oswald, F., Lopes, A., Skoda, K., Hesse, C. L., \& Pedersen, C. L. (2020). I'll show you mine so you'll show me yours: Motivations and personality variables in photographic exhibitionism. Journal of Sex Research, 57(5), 597-609.

Paasonen, S., Light, B., \& Jarrett, K. (2019). The dick pic: Harassment, curation, and desire. Social Media + Society, 5(2), 1-10.

Pingel, E. S., Bauermeister, J. A., Johns, M. M., Eisenberg, A., \& Leslie-Santana, M. (2013). "A safe way to explore" reframing risk on the internet amidst young gay men's search for identity. Journal of Adolescent Research, 28(4), 453-478.

Powell, A., \& Henry, N. (2017). Sexual violence in a digital age. Springer.

Race, K. (2015). Speculative pragmatism and intimate arrangements: Online hook-up devices in gay life. Culture, Health \& Sexuality, 17(4), 496-511.

RÉZO (2021). Le consentement entre hommes? Oui. https://www.rezosante.org/consentement/

Ross, L., Greene, D., \& House, P. (1977). The "false consensus effect": An egocentric bias in social perception and attribution processes. Journal of Experimental Social Psychology, 13(3), 279-301.

Simon, W., \& Gagnon, J. H. (2003). Sexual scripts: Origins, influences and changes. Qualitative Sociology, 26(4), 491-497.

Smith, J. A., Flowers, P., \& Larkin, M. (2009). Interpretative phenomenological analysis: Theory, method and research. SAGE.

Snell, W. E., Jr., Hawkins, R. C., \& Belk, S. S. (1988). Stereotypes about male sexuality and the use of social influence strategies in intimate relationships. Journal of Social and Clinical Psychology, 7(1), $42-48$.

Sweeney, B. N. (2014). Sorting women sexually: Masculine status, sexual performance, and the sexual stigmatization of women. Symbolic Interaction, 37, 369-390.

Tinder (n.d.). Dating safety tips. https://policies.tinder.com/safety/intl/en

Tziallas, E. (2015). Gamified eroticism: Gay male "social networking" applications and self-pornography. Sexuality and Culture, 19(4), 759-775.

Vitis, L., \& Gilmour, F. (2017). Dick pics on blast: A woman's resistance to online sexual harassment using humour, art and Instagram. Crime, Media, Culture: An International Journal, 13(3), 335-355.

Waldman, A. E. (2019). Law, privacy, and online dating: "Revenge porn" in gay online communities. Law \& Social Inquiry, 44(4), 987-1018.

Waling, A., Kerr, L., Bourne, A., Power, J., \& Kehler, M. (2020). 'It's nice to be appreciated': Understanding heterosexual men's engagements with sexting and sharing Dick Pics. Sexualities, 1-24.

Waling, A., \& Pym, T. (2017). 'C'mon, No One Wants a Dick Pic': Exploring the cultural framings of the 'Dick Pic' in contemporary online publics. Journal of Gender Studies, 28(1), 70-85.

Yeung, T. H., Horyniak, D. R., Vella, A. M., Hellard, M. E., \& Lim, M. S. (2014). Prevalence, correlates and attitudes towards sexting among young people in Melbourne, Australia. Sex Health, 11(4), 332-339.

Publisher's Note Springer Nature remains neutral with regard to jurisdictional claims in published maps and institutional affiliations. 